IP Periodica Polytechnica Civil Engineering

61(1), pp. 154-163, 2017

DOI: $10.3311 /$ PPci.10214

Creative Commons Attribution (i)

RESEARCH ARTICLE

\section{Robust Topology Optimization: A New Algorithm for Volume-constrained Expected Compliance Minimization with Probabilistic Loading Directions using Exact Analytical Objective and Gradient}

\author{
Anikó Csébfalvi $1^{*}$
}

Received 22 June 2016; accepted 16 October 2016

\begin{abstract}
This paper presents a new algorithm for volume-constrained expected compliance minimization of continuum structures with probabilistic loading directions using analytically determined exact objective and gradient functions. The algorithm is based upon the finding that for a particular set of statistical parameters the integration in the expected compliance function can be done symbolically and automatically using symbolic manipulation software. In this study, Mathematica was used to integrate and simplify the highly nonlinear expected compliance function. It will be demonstrated by examples that the result of the symbolic pre-processing step is a simple linear function defined on a particular subset of the inverse stiffness entries which is needed in the compliance computation. The coefficient set of this linear function forms the base of the exact analytical gradient computation used in the optimal solution searching optimality criteria (OC) method to define the steepest descent direction. Naturally, the applied OC method can be replaced by any other appropriate nonlinear solver. Matlab codes of the algorithm for $2 D$ and $3 D$ structures with exact analytical sensitivities have been developed using the topology optimization codes presented by Andreassen et al. [1] for $2 D$ and Liu and Tovar [2] for 3D structures as starting points. Illustrative examples with Mathematica and Matlab codes are presented to demonstrate the essence and viability of the proposed approach and highlight the potential of the automatic symbolic computation in structure optimization.
\end{abstract}

\section{Keywords}

topology optimization, uncertain parameters, probabilistic load direction, expected compliance, symbolic computation

${ }^{1}$ University of Pécs, Hungary

${ }^{*}$ Corresponding author email: csebfalvi.witch@gmail.com

\section{Introduction}

In the real-world topology optimization problems, the optimal performance obtained using conventional deterministic methods can be dramatically degraded in the presence of sources of uncertainty. The source of uncertainty may be the variability of applied loads, spatial positions of nodes, material properties, and so on. Various deterministic and stochastic approaches have been developed to account for different types of uncertainty in structural design and optimization methods to get robust and reliable solutions. The interested reader is directed to Bendsøe and Sigmund [3] and Deaton and Grandhi [4] which contain extensive bibliographies on this subject. In this paper, it is assumed that the only source of uncertainty is the variability of the applied load directions and the compliance is used as performance measure in the in the volume-constrained topology optimization. The volume-constrained structural optimization models of continuum structures which are able to take into account the directional uncertainty of the applied loads can be divided into two groups [5]: (1) deterministic and (2) stochastic. A critical examination and comparison of the volumeconstrained deterministic and stochastic topology optimization models with uncertain load directions, from engineering point of view, was presented by Csébfalvi and Lógó [6].

The deterministic models which try to minimize the volume-constrained worst compliance on the set of feasible loading directions can be formulated by several different ways. De Gournay et al. [7] presented an approach for shape and topology optimization of the robust compliance via the level set method which minimizes the worst-case compliance using a semi-definite programming method to select the best descent direction in the iteration process. Thore et al. [8] presented a large-scale robust topology optimization method under loaduncertainty where the loads vary in uncertainty sets. The problem can be formulated as a semi-infinite optimization problem, which can be replaced by a non-linear semi-definite problem. A worst-load-direction oriented unified common framework was presented by Csébfalvi [9] for robust optimization of both continuum and truss structures with uncertain load directions, which can be used for volume minimization of continuum 
structures with compliance constraints and weight minimization of truss structures with displacement and stress constraints.

The stochastic models apply parametric statistical tools to describe the directional uncertainty of the loads. The most popular model minimizes the volume-constrained expected compliance with loading directional uncertainty, where the directional uncertainties are assumed normally distributed and statistically independent. The expected compliance minimization model can be transformed to a standard volumeconstrained multi-load compliance-minimization problem by several different ways where the load cases and weights are derived analytically or numerically. However, it is not straightforward how could be select the load cases to ensure that all critical cases are considered in the sampling schema which needed to obtain an accurate approximation of the original continuous problem. Naturally, the discretization of an originally continuous problem always means simplification which should be avoided as much as possible. Dunning et al. [10] proposed a "pseudo-sample-based" stochastic method for considering loading directional uncertainty in topology optimization in order to produce robust solutions. In the volume-constrained expected compliance minimization problem the uncertainties are described by statistically independent continuous normal probability functions. Starting from the exact expected compliance function after lengthy symbolic manipulation and simplification, the authors get a model which is analogous to a multiload topology optimization problem where the load cases and their weights are derived analytically. Lógó [11] presented a new type of probabilistic optimal topology design method for continuum type of structures where the points of application of the loads are given randomly. In the proposed probabilistic topology optimization method the minimum penalized weight design of the discretized structure is subjected to compliance constraint and side constraints. The compliance expression is probabilistic one. Carrasco et al. [12] presented a samplebased approach for the volume-constrained expected compliance minimization in topology optimization with stochastic load directions. In this paper, it was shown that the compliance minimization problem can be transformed into a standard multi-load topology optimization problem in which the loading scenarios are related to the variance of the random main load. Liu and Wen [13] presented a topology optimization model for continuum structures with uncertainty in loading direction. In their approach, the original stochastic volume-constrained expected compliance minimization problem was replaced by a standard volume-constrained deterministic multi-load problem, where the load cases are derived to accurately compute the expected compliance. The loading directional uncertainties were described by interval variables without considering their distributions, which in the sampling process were approximated by their deterministic midpoints. Guo et al. [14] presented a multi-scale robust concurrent optimization framework for varying material structure with unknown-but-bounded load uncertainties modelled by well-known ellipsoid model.

Similarly to the Dunning et al. [10] model, the presented new approach is also based on the exact analytical form of the expected compliance function but it exploits the finding that when the numerical values of the statistical parameters of the directional uncertainties are known then the highly nonlinear expected compliance minimization problem can be solved as a volume-constrained single-load topology optimization problem where the single load is the mean load. In the new model the objective is the exact analytical expected compliance function in an extremely simple form generated symbolically and automatically using symbolic manipulation software. In the expected compliance minimization process exact analytical sensitivities can be used to define the steepest descent direction. The paper is organized as follows. Section 2 focuses on the mathematical formulation of the considered problem. The examples used to illustrate the proposed approach are presented in Section 3. Finally, some concluding remarks are presented in Section 4.

\section{The proposed exact approach}

The traditional deterministic 2D topology optimization problem can be formulated as follows:

$$
\begin{gathered}
c(x)=U^{\prime} K U \rightarrow \min \\
V(x)=\varphi V_{0} \\
K U=F \\
0 \leq x \leq 1
\end{gathered}
$$

where $\mathrm{x}$ is the matrix of design variables (the element densities), $\mathrm{c}(\mathrm{x})$ is the compliance, $\mathrm{U}$ and Fare the global displacement and load vectors, respectively, $\mathrm{K}$ is the global stiffness matrix, $\mathrm{V}(\mathrm{x})$ and $\mathrm{V} 0$ are the material volume and design domain volume, respectively, and $\varphi$ is the prescribed volume fraction. The design domain is assumed to be rectangular and discretized with $\mathrm{n}=\mathrm{ex} \times$ ey square elements with four nodes per element and two degrees of freedoms (DOFs) per node: $x=\left\{x_{i j} \mid j \epsilon\right.$ $\left\{1,2, \ldots, e^{y}\right\}, i \in\left\{1,2, \ldots, e^{x}\right\}$. Both nodes and elements are numbered column-wise from left to right, and the DOFs $2 i-1$ and $2 i$ correspond to the horizontal and vertical displacements of node $i, i \in\{1,2, \ldots, 2 n\}$, respectively. The optimization problem (1-4) can be solved by, for example, the well-known optimality criteria (OC) method.

For sake of simplicity and without loss of generality the proposed exact approach will be described only for 2D structures with only one load with magnitude $f$ and normally distributed loading direction which is characterized by the mean direction $\mu$ and standard deviation $\sigma$. In Figure 1, the essence of the applied probabilistic approach is shown, where the first two dimensions define the 2D angle set around the nominal load direction and 
the third dimension describes the normal probability density function normal $(\mu, \sigma, \alpha)$ in the $\mu-3 \sigma \leq \alpha \leq \mu+3 \sigma$ interval where the mean direction is $\mu=2 \pi / 3$ and the standard deviation as the measure of the spreading around the mean direction is $\sigma=\pi / 18$. According to the well-known three-sigma-rule the angle set describes the so-called "natural fluctuation" of the load directions around the mean (nominal) direction with

$$
\int_{\mu-3 \sigma}^{\mu+3 \sigma} \operatorname{normal}(\mu, \sigma, \alpha) d \alpha=0.9973
$$

probability which can be approximated by one. A single 2D point load with magnitude $f$ which is acting in an arbitrary direction can be written in terms of two orthogonal loads according to the horizontal $x$ and the vertical $y$ directions:

$$
\begin{aligned}
& f_{x}=f \cos (\alpha) \\
& f_{y}=f \sin (\alpha)
\end{aligned}
$$

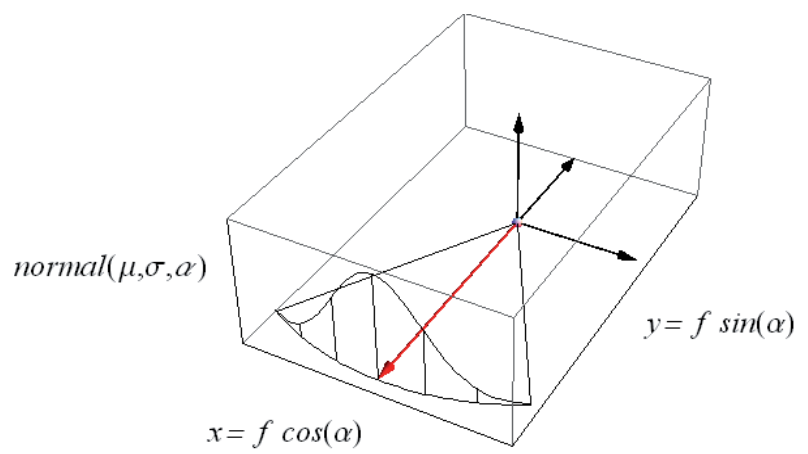

Fig. 1 Natural fluctuation of the nominal load direction with $\pm \mu / 6$ loading directional uncertainty with 0.9973 probability

Firstly, we define the expected-compliance function $\overline{\mathrm{c}}$ in exact analytical form:

$$
\bar{c}(x)=\int_{\mu-\pi}^{\mu+\pi} F(\alpha)^{\prime} K^{-1} F(\alpha) p d f(\mu, \sigma, \alpha) d \alpha
$$

where the $\mathrm{m}=2 \times\left(e^{x}+1\right) \times\left(e^{y}+1\right)$ dimensional sparse load vector $F(\alpha)$ has at least one and at most two non-zero entries in the function of $\alpha$ and the integration limits are set according to the full circle of $2 \pi$.

The volume constrained expected compliance minimization problem can be written in the following form:

$$
\begin{gathered}
\bar{c}(x)=\int_{\mu-\pi}^{\mu+\pi} F(\alpha)^{\prime} K^{-1} F(\alpha) p d f(\mu, \sigma, \alpha) d \alpha \rightarrow \min \\
V(x)=\varphi V_{0} \\
K U=F \\
0 \leq x \leq 1
\end{gathered}
$$

where $\mathrm{x}$ is the matrix of design variables (the element densities), $\bar{c}(\mathrm{x})$ is the expected-compliance function, $\mathrm{U}$ and $\mathrm{F}$ are the nominal displacement and load vectors, respectively, $\mathrm{K}$ is the global stiffness matrix, $\mathrm{V}(\mathrm{x})$ and V0 are the material volume and design domain volume, respectively, and $\varphi$ is the presetting volume fraction. We have to note it again that when we know the numerical values of the statistical parameters then after symbolic integration and simplification we can eliminate the statistical parameters from the objective function. Therefore the result will be a standard nonlinear optimization problem which can be solved by several different ways. The result of the optimization will be a robust expected-complianceminimal design for the prescribed $\varphi$ volume fraction. It will be demonstrated by examples in Section 3 that the result of the symbolic pre-processing step will be a simple linear function defined on a particular subset of the inverse stiffness entries which is needed in the compliance computation. As we are only interested in linear elastic structures, the stiffness matrix $K$ and its inverse $K^{-1}$ are symmetrical. For the numerical treatment of the examples presented in Section 3 the standard OC method was used with exact analytical sensitivities: $\partial \bar{c}(x) / \partial x$.

\section{Examples}

In this section, we present two numerical examples with uncertain loading direction to demonstrate the efficiency and simplicity of the exact analytical objective function formulation. The first example is a simple symmetric "academic" twodimensional (2D) problem with one uncertain load for which in the case of trusses, as a function of side loads, the analytical solutions are known. The second example is a three-dimensional (3D) analogy of the first one.

It will show that when the numerical value of the mean and standard deviation is known then the simplified exact analytical form of the expected compliance function $\bar{c}(x)$ can be generated symbolically and automatically. To the best of our knowledge this result is new in the literature. In this study, for the symbolic computation the state-of-art Mathematica package was used. In its simplified form the expected compliance is a linear function of a $2 \times 2(2 \mathrm{D})$ or $3 \times 3(3 \mathrm{D})$ dimensional $Q$ matrix which consists of the entries of $K^{-1}$ which are needed in compliance computation. It is important to note that $Q$ can be generated without computing the full inverse of $K$ which, from computational point of view, a very important result. For example, in Matlab the following simple code can be used to select column $C_{j}$ from the inverse $S^{-1}$ of an $n \times n$ symmetric matrix $\mathrm{S}: \mathrm{W}=$ zeros $(\mathrm{n}, 1) ; \mathrm{W}(\mathrm{j})=1 ; \mathrm{C}=\mathrm{S} / \mathrm{W}$;

The generated simple form of the expected-compliance can be used as the starting base of the new SIMP-type expected compliance minimization algorithm. Matlab codes of the algorithm for 2D and 3D structures can be developed with straightforward modifications from the topology optimization codes presented by Andreassen et al. [1] for 2D and Liu and Tovar [2] for 3D structures. Each code has been developed using the 99 line code presented by Sigmund [15] as a starting point. It 
will be demonstrated in a forthcoming paper that the presented approach based on the symbolic pre-processing can be generalized to handle more than one uncertain loads without any difficulties because replacing the trigonometric functions with their complex exponential forms the argument of the multiple integral will become an "integration-friendly" expression and the number of the nonzero entries of the load vector is very low comparing it with the load vector size.

In the following, it will be shown that $\partial K^{-1} / \partial x_{j i}$, where $j$ $\epsilon\left\{1,2, \ldots, e^{y}\right\}$ and $i \in\left\{1,2, \ldots, e^{x}\right\}$ can be expressed in the following form:

$$
\partial K^{-1} / \partial x_{i j}=-K^{-1} \partial K / \partial x_{i j} K^{-1}
$$

Starting with the definition of the inverse

$$
K^{-1} K=I
$$

and differentiate, yielding

$$
K^{-1} \partial K / \partial x_{j i}+\partial K^{-1} / \partial x_{j i} K=0
$$

rearranging the terms yields

$$
\partial K^{-1} / \partial x_{j i}=-K^{-1} \partial K / \partial x_{j i} K^{-1}
$$

According to the above expression the load specific derivative entries $\partial Q / \partial x_{j i}$ can be generated by simple matrix manipulations. In the next sections, the Matlab codes for the presented 2D and 3D examples well-illustrate the essence of the sensitivity generating process. In the Matlab codes the readability was preferred against the computational efficiency. It has to be note that the presented exact algorithm and the correctly described benchmark problems with reproducible numerical results may be used for testing the quality of new exact and heuristic solution procedures to be developed in the future for structural optimization with varying load directions.

\subsection{A 2D example}

In this section, a simple "academic" $2 \mathrm{D}$ example is presented to illustrate the essence of the proposed new volume constrained expected compliance minimization topology optimization approach. The example, shown in Figure 2, is a cantilever beam, with a ground structure of $40 \times 80$ rectangular elements and an external load $f$ is acting in the end-middle position of the beam and its value is $f=-10$ and its mean (nominal) direction is $\mu=0$ and the standard deviation as the measure of the spreading around mean direction is $\sigma=\pi / 18$. Applying the well-known three-sigma rule, the "natural fluctuation" of the uncertain direction will be $\pm \pi / 6$ with probability 0.9973 . The Young's modulus is $E_{0}=1$, the Poisson's ratio is $v=0$ and the fixed volume fraction is $\varphi=0.2$. The penalization power is $p=$ 3 and sensitivity filtering is applied with filter radius $r_{\text {min }}=1.5$.

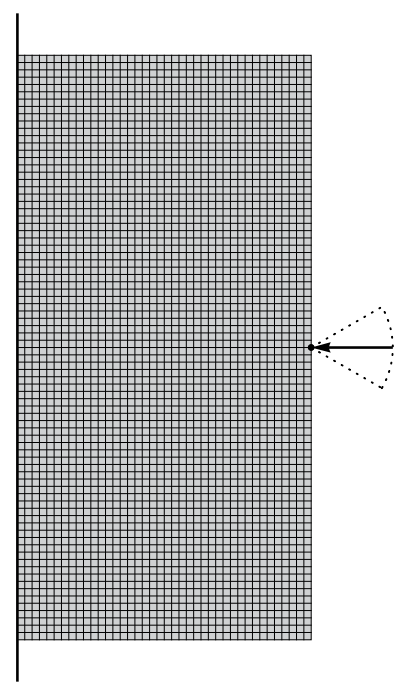

Fig. 2 The design domain, boundary conditions, and the external load with $+\pi$ / 6 "natural fluctuation" of a cantilever beam

The problem-specific expected compliance function $\bar{c}$ in its exact analytical form will be the following:

$$
\begin{aligned}
& \bar{c}=\int_{\mu-\pi}^{\mu+\pi}[f \cos (\alpha) f \sin (\alpha)]\left[\begin{array}{ll}
Q_{x x} & Q_{x y} \\
Q_{x y} & Q_{y y}
\end{array}\right]\left[\begin{array}{l}
f \cos (\alpha) \\
f \sin (\alpha)
\end{array}\right] \\
& \times \operatorname{normal}(\mu, \sigma, \alpha) d \alpha
\end{aligned}
$$

where normal $(\mu, \sigma, \alpha)$ is the normal density function and $Q=\left[\begin{array}{ll}Q_{x x} & Q_{x y} \\ Q_{x y} & Q_{y y}\end{array}\right]$ is a symmetric $2 \times 2$ matrix consists of the entries of $K^{-1}$ needed in the compliance computation and the integration limits are set according to the full circle of $2 \pi$. The Mathematica code of the symbolic pre-processing step is presented in Figure 3.

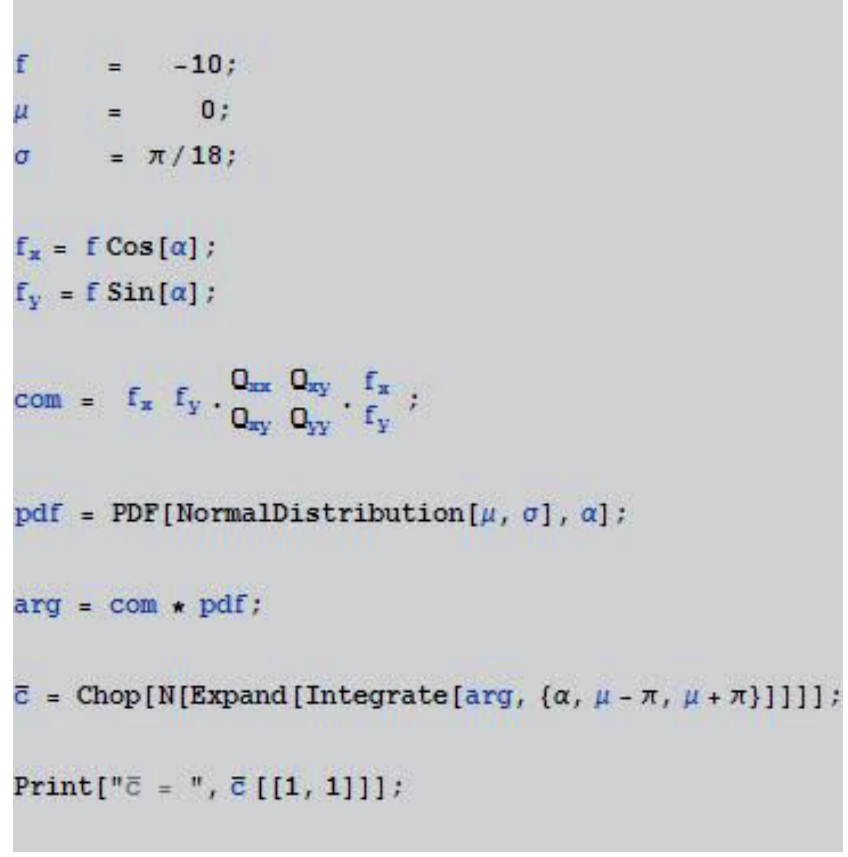

Fig. 3 The Mathematica code of the symbolic pre-processing step 
The result will be an extremely simple expression for $\overline{\mathrm{c}}$ :

$$
\bar{c}=97.0448 Q_{x x}+2.95524 Q_{y y}
$$

In this example, our goal is the following: we try to find an expected compliance minimal robust solution for the fixed volume fraction $\varphi=0.2$. The design domain, boundary conditions, and the external load with $\pm \pi / 6$ "natural fluctuation" of the cantilever beam is shown in Figure 3.

The nominal compliance minimal shape with $\alpha=0$, and the expected compliance minimal shape with $\sigma=\pi / 18$ directional uncertainty are shown in Figure 4-5, respectively. Let $\overleftarrow{c}(x)$ and $\vec{c}(x)$ define the minimal and maximal compliance value function, respectively. The range function $\vec{c}(x)$ is defined as $\vec{c}(x)=\vec{c}(x)-\bar{c}(x)$. The performance measures using the nominal and expected compliance minimization models are presented in Table 1. In Figure 6, we show the nominal and expected compliance values on the set of the feasible load directions with $\sigma=\pi / 18$ directional uncertainty using the nominal and expected compliance-minimizing models. The nominal and expected compliance values are represented by solid and dashed lines, respectively. The robust optimization process changes the nominal shape drastically. Figure 6 well-demonstrate the smoothing effect of the expected compliance model.

Table 1 Performance measures

\begin{tabular}{ccccc}
\hline model & $\bar{c}(x)$ & $\bar{c}(x)$ & $\vec{c}(x)$ & $\vec{c}(x)$ \\
\hline$c \rightarrow \min$ & 610.38 & 441.48 & 1870.30 & 1428.82 \\
$\bar{c} \rightarrow \min$ & 551.60 & 480.29 & 1083.52 & 603.23 \\
\hline
\end{tabular}

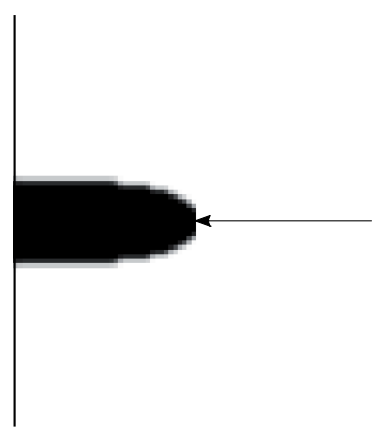

Fig. 4 Nominal compliance minimal shape with $\alpha=0$

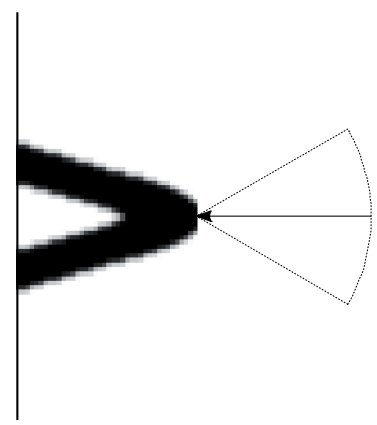

Fig. 5 Expected compliance minimal shape with $\sigma=\pi / 18$ directional uncertainty

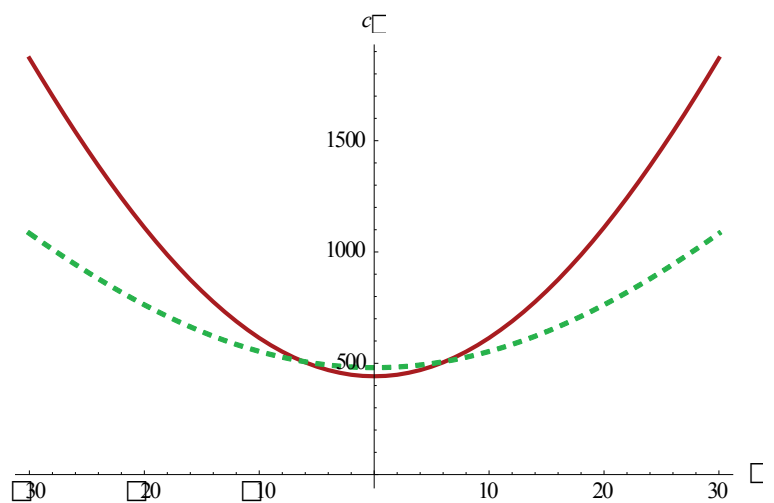

Fig. 6 The compliance values with $\sigma=\pi / 18$ directional uncertainty using the nominal and expected compliance minimizing models

The Matlab code of the volume constrained expected compliance minimization problem can be given by straightforward modifications from the top $88 . \mathrm{m}$ program developed by Andreassen et al. [1] for $2 \mathrm{D}$ continuum structures. In the program, only two labelled code sections have to be modified to get a code for the volume constrained expected compliance minimization. The first code section which is labelled by "응 DEFINE LOADS AND SUPPORTS ..." defines the loads and supports and the second section labelled by "\% OBJECTIVE FUNCTION AND SENSITIVITY ANALYSIS" defines the objective function and its sensitivities. The problem-specific code section replacements are the followings:

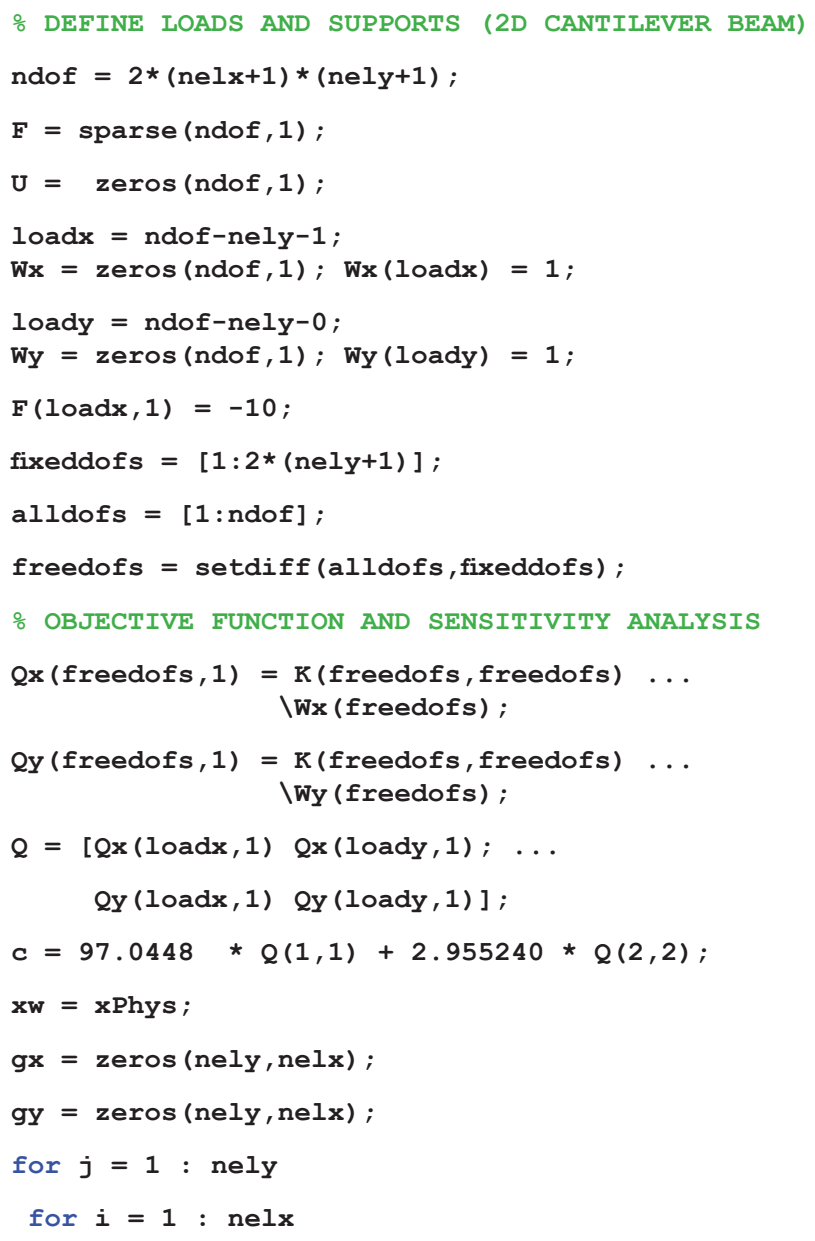




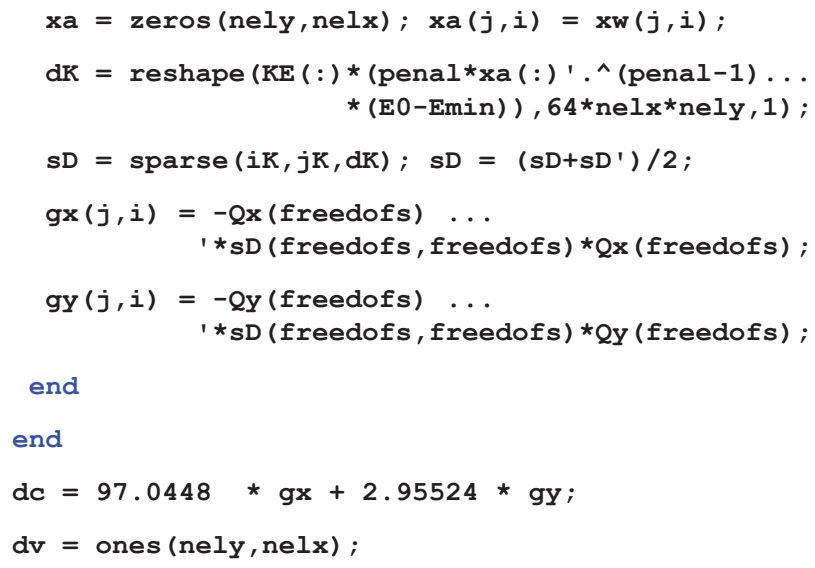

\subsection{A 3D example}

In this section, a simple $3 \mathrm{D}$ problem is presented as a three-dimensional analogy of the previously investigated 2D problem. The example shown in Figure 7, is a cantilever beam, with a ground structure of $20 \times 10 \times 10$ hexahedral elements and an external load $f$ is acting in the end-middle-middle position of the beam and its value is $f=-1$ and its nominal direction is orthogonal to the $y-z$ plane.

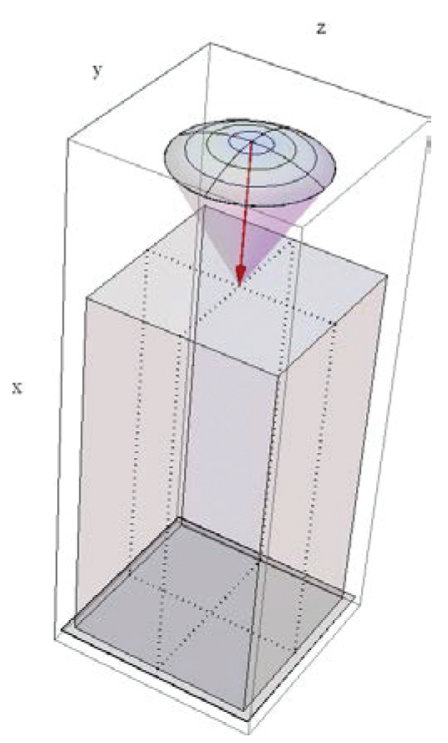

Fig. 7 The design domain, boundary conditions, and external load with directional uncertainty of the $3 \mathrm{D}$ cantilever beam

The Young's modulus is $E_{0}=1$, the Poisson's ratio is $v=$ 0 , and the fixed volume fraction is $\varphi=0.25$. The penalization power is $p=3$ and sensitivity filtering is applied with filter radius $r_{\min }=1.5$. First, as a straightforward 3D extension of the previously investigated 2D symmetric loading scenario, it is assumed that the random directional perturbations of the external point load form a symmetric 3D spherical region around the nominal load direction which can be described by an angle set $\{\alpha, \beta\}$, where $\alpha, 0 \leq \alpha \leq 2 \pi$, follows a uniform distribution on the full circle of $2 \pi$ and $\beta$ follows a normal distribution with mean $\mu=0$ and standard deviation $\sigma=\pi / 18$, where angle $\alpha$ defines the perturbed load direction in the $y-z$ plane from the fixed loading point and angle $\beta$ defines the declination from the nominal load direction with $-\pi / 6 \leq \beta \leq+\pi / 6$ "natural fluctuation" according to the three-sigma rule. Now, using the previously defined particular statistical parameter set, the problem-specific exact expected-compliance function $\overline{\mathrm{c}}$ can be defined in the following analytical form:

$$
\begin{aligned}
& \bar{c}=\int_{\mu-\pi}^{\mu+\pi} \int^{2 \pi 0} c(\alpha+\beta) \times \operatorname{normal}(\mu, \sigma, \alpha) \times \\
& \text { uniform }(\underline{\alpha}, \bar{\alpha}, \alpha) d \alpha d \beta
\end{aligned}
$$

where

$$
c(\alpha, \beta)=\left[f_{x}(\beta) f_{y}(\alpha, \beta) f_{z}(\alpha, \beta)\right] Q\left[\begin{array}{l}
f_{x}(\beta) \\
f_{y}(\alpha, \beta) \\
f_{z}(\alpha, \beta)
\end{array}\right]
$$

is the compliance $c(\alpha, \beta)$ as the function of angle set $\{\alpha, \beta\}$, $Q$ is a $3 \times 3$ matrix consists of the entries of $K^{-1}$ which are needed in compliance computation, and the density function $p d f(\alpha, \beta)$ of $c(\alpha, \beta)$ is a product of a normal and a uniform density functions according to the statistical independency:

$$
\begin{gathered}
f_{x}(\beta)=f \cos (\beta) \\
f_{y}(\alpha, \beta)=f \sin (\beta) \cos (\alpha) \\
f_{z}(\alpha, \beta)=f \sin (\beta) \cos (\alpha) \\
Q=\left[\begin{array}{lll}
Q_{x x} & Q_{x y} & Q_{x z} \\
Q_{x y} & Q_{y y} & Q_{y z} \\
Q_{x z} & Q_{y z} & Q_{z z}
\end{array}\right] \\
p d f=(\alpha, \beta)=\text { normal }(\mu, \sigma, \beta) \times \text { uniform }(\underline{\alpha}, \bar{\alpha}, \alpha)
\end{gathered}
$$

In the exact formulation the outer integration limits are taken to be $\mu \pm \pi$, which integrates over the full revolution of $2 \pi$.

The Mathematica code of the symbolic pre-processing step is presented in Figure 8. 


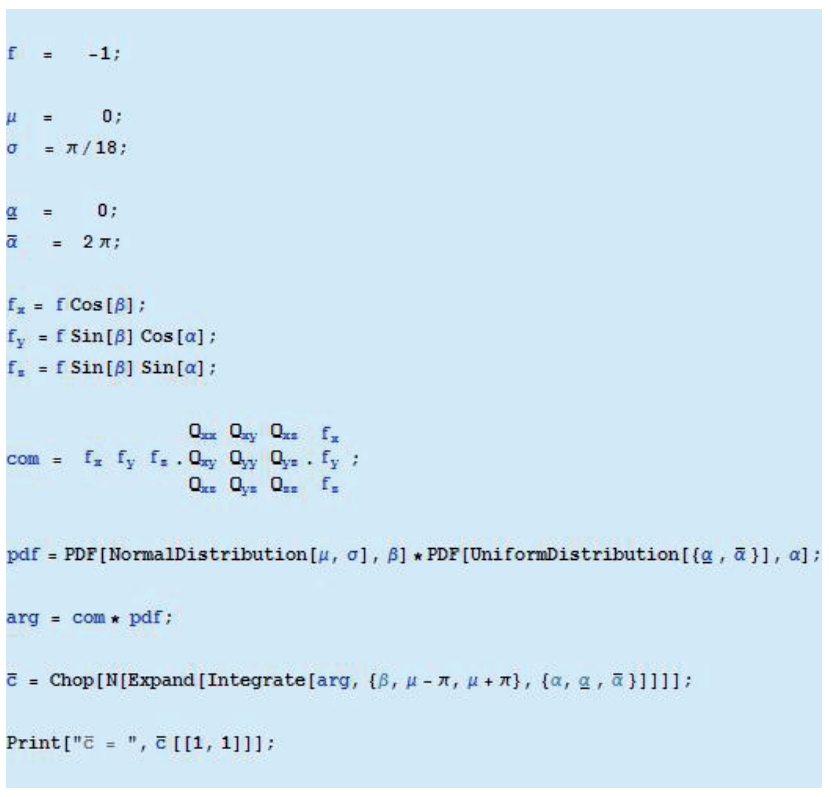

Fig. 8 The Mathematica code of the symbolic pre-processing step

After automatic symbolic integration and simplification the result will be the following extremely simple expression for $\bar{c}$ :

$$
\bar{c}=0.970448 Q_{x x}+0.0147762 Q_{y y}+0.0147762_{z z}
$$

Note that in the expression of $\bar{c}$ the coefficients of $Q_{y}$ and $Q_{z}$ are exactly the same because the expected compliance values form a circular shape around the loading point because the loading condition is centrally-symmetric in the $y-z$ plane. The uncertain loading scenario is presented in Figure 9. The nominal compliance minimal shape and the expected compliance minimal shape with $\sigma=\pi / 18$ spherical directional uncertainty are shown in Figure 10-11, respectively. The performance measures using the nominal and expected compliance minimization models are presented in Table 2. In Figure 12-13, the nominal and expected compliance values are shown on the set of the feasible load directions with $\beta= \pm 3 \times \pi / 18$ spherical directional perturbation using the nominal and expected compliance minimizing models. In Figure 14, for the reason of easy visual comparison we show the unified plot of the results of the nominal and expected compliance models on the "natural" subset of the spherical directional perturbations. The expected compliance minimization model results in a more balanced shape than the nominal compliance minimization model. In other words, a relatively small change in the expected compliance values may change the compliance shapes drastically.

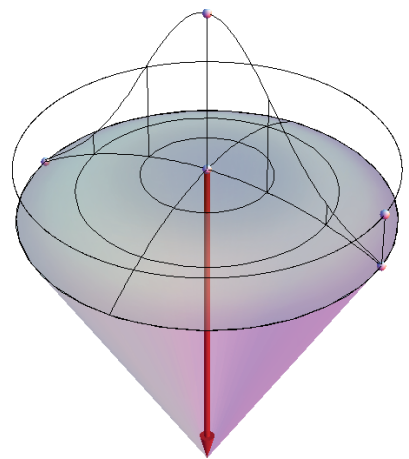

Fig. 9 The loading scenario

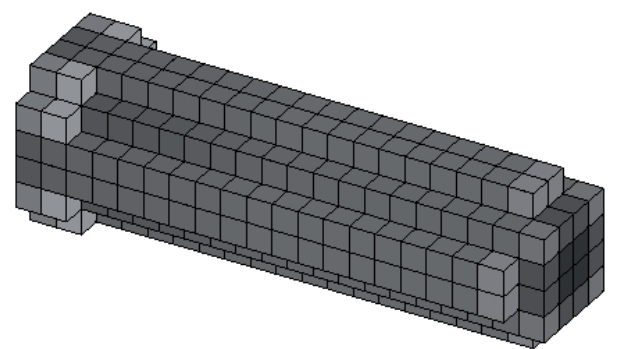

Fig. 10 The nominal compliance minimal shape of the 3D cantilever beam

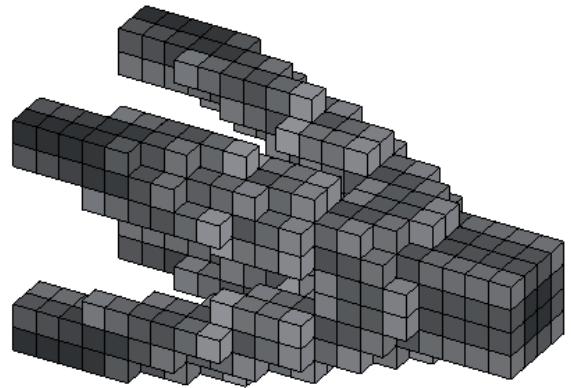

Fig. 11 The expected compliance minimal shape of the 3D cantilever beam

Table 2 Performance measures for the compliance and exact expected compliance minimizing models

\begin{tabular}{ccccc}
\hline model & $\bar{c}(x)$ & $\bar{c}(x)$ & $\vec{c}(x)$ & $\vec{c}(x)$ \\
\hline$c(x) \rightarrow \min$ & 4.8991 & 2.4835 & 22.9184 & -20.4349 \\
$\bar{c}(x) \rightarrow \min$ & 4.0723 & 3.2490 & 10.2138 & -6.9648 \\
\hline
\end{tabular}

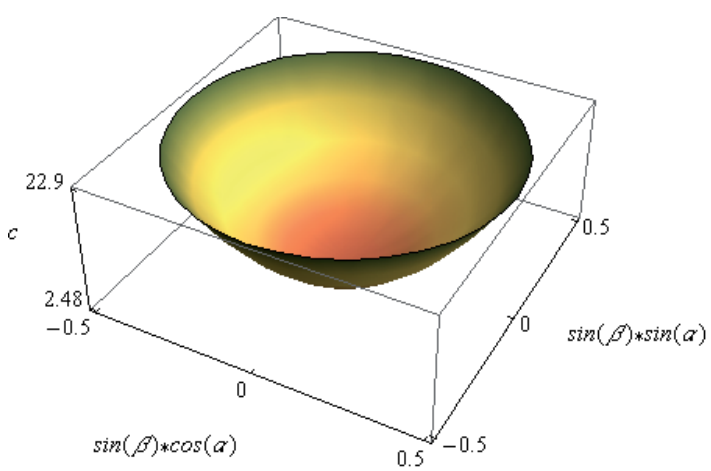

Fig. 12 The compliances of the 3D cantilever beam on the set of the spherical direction perturbations using the nominal compliance minimal solution 


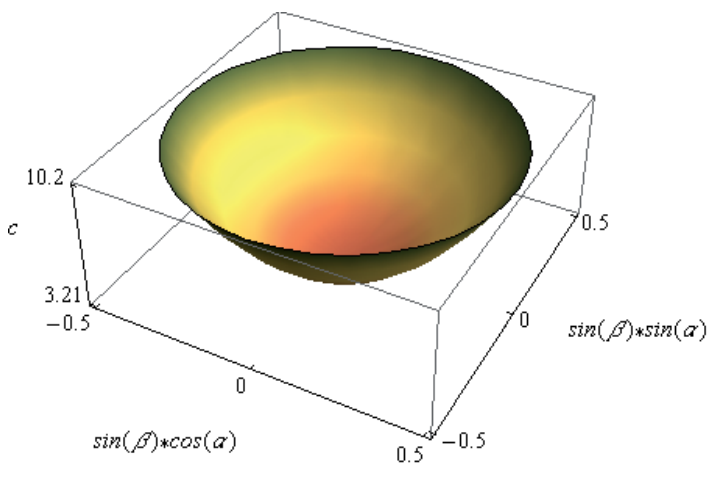

Fig. 13 The compliances of the 3D cantilever beam on the set of the spherical direction perturbations using the expected compliance minimal solution

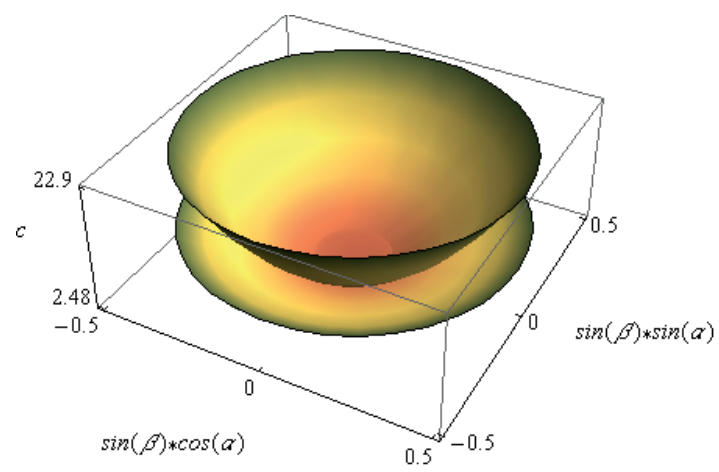

Fig. 14 The visual comparison of the compliances given by the nominal and expected compliance minimizing models on the set of the spherical direction perturbations

Now, to demonstrate the flexibility and general applicability of the proposed exact solution searching process, the previously investigated $3 \mathrm{D}$ problem will be solved with two other stochastic loading scenarios. In each case, angle $\alpha$ follows a normal distribution with mean $v=0$ and standard deviation $\tau=$ $\pi / 18$. In the first scenario, angle $\beta$ follows a normal distribution with mean $\mu=0$ and standard deviation $\sigma=\pi / 18$ which, in the second scenario, is replaced by a truncated normal distribution where the feasible angle set $\beta \leq \beta \leq \bar{\beta}$ is defined by the 0 $\leq \beta \leq \pi / 6$ relation. The automatically generated exact objective functions, in the description order, are the followings:

$$
\begin{aligned}
& \bar{c}=0.970448 Q_{x x}+0.028679 Q_{y y}+0.000873343 Q_{z z} \\
& \bar{c}=0.970448 Q_{x x}+0.028679 Q_{y y}+0.000873343 Q_{z z} \\
& +0.26343 Q_{x y}
\end{aligned}
$$

The performance measures are presented in Table 3. The loading scenarios, the probability density functions, and the exact optimal shapes are visualized in Figure 15 and 18, Figure 16 and 19, and Figure 17 and 20, respectively. The presented loading scenarios drastically change the optimal shape of the $3 \mathrm{D}$ cantilever beam.
Table 3 Performance measures of the investigated loading scenarios

\begin{tabular}{ccc}
\hline $\boldsymbol{p d f}(\boldsymbol{\alpha}, \boldsymbol{\beta})$ & $\boldsymbol{c}(\boldsymbol{x})$ & $\overline{\boldsymbol{c}}(\boldsymbol{x})$ \\
\hline normal $(v, \tau, \alpha) \times$ normal $(\mu, \sigma, \beta)$ & 2.7150 & 3.3191 \\
$\operatorname{normal}(v, \tau, \alpha) \times \stackrel{\text { normal }}{\longrightarrow}(\mu, \sigma, \underline{\beta}, \bar{\beta}, \beta)$ & 2.9542 & 2.9867 \\
\hline
\end{tabular}

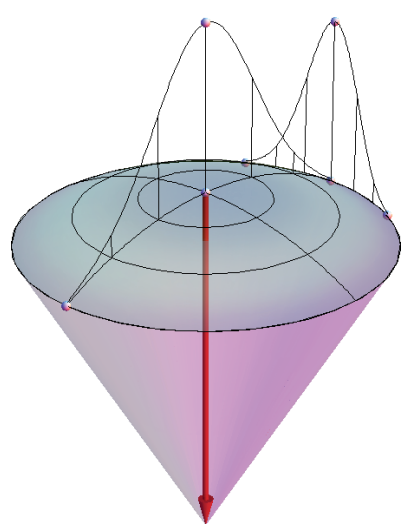

Fig. 15 The $p d f(\alpha, \beta)=$ normal $(v, \tau, \alpha) \times \operatorname{normal}(\mu, \sigma, \beta)$ loading scenario

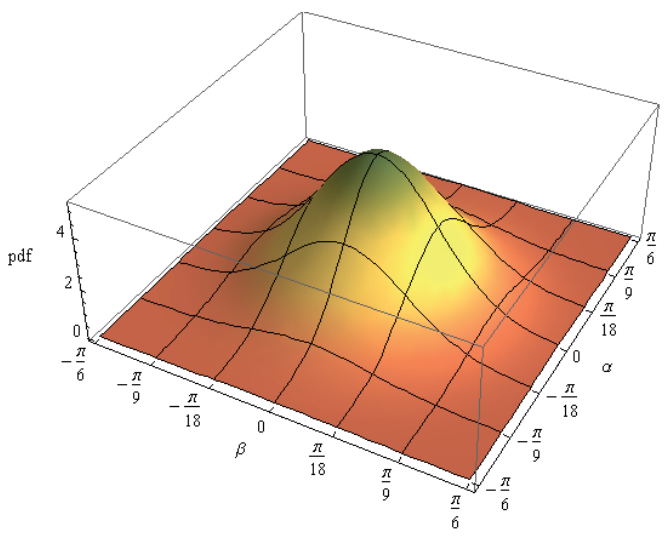

Fig. 16 The shape of $p d f(\alpha, \beta)$

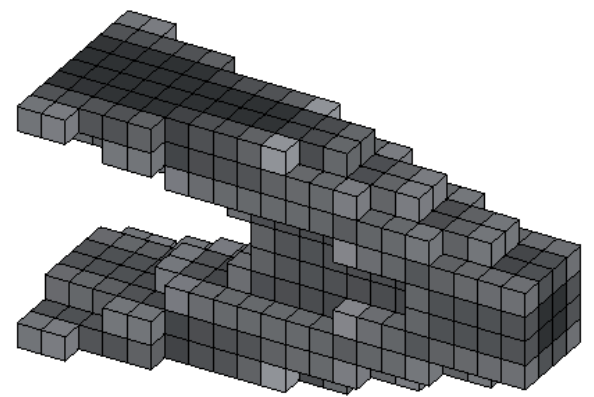

Fig. 17 The exact optimal shape of the 3D cantilever beam 


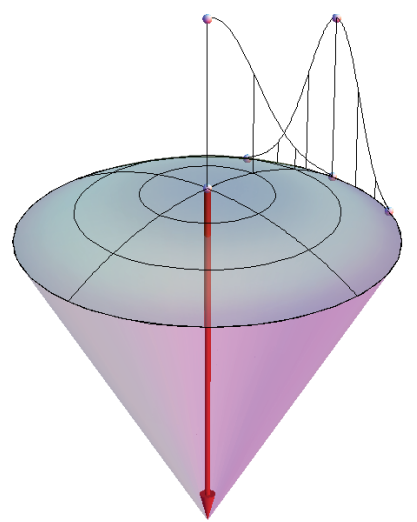

Fig. 18 The $p d f(\alpha, \beta)=\operatorname{normal}(v, \tau, \alpha) \times \stackrel{\text { normal }}{\longrightarrow}(\mu, \sigma, \underline{\beta}, \bar{\beta}, \beta)$ loading scenario

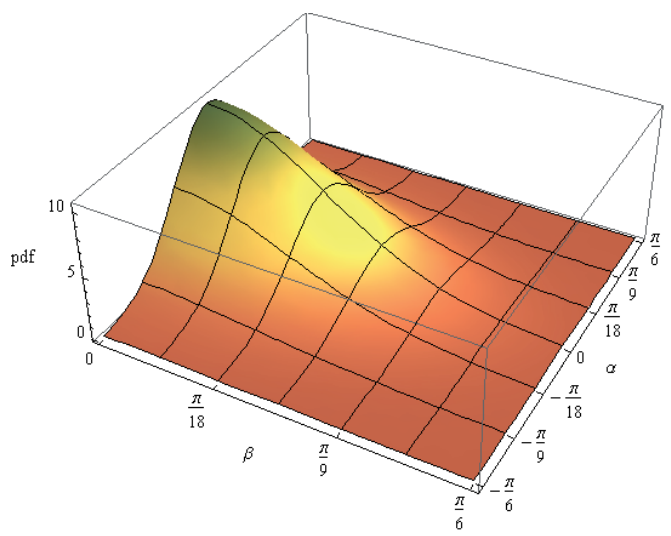

Fig. 19 The shape of $p d f(\alpha, \beta)$

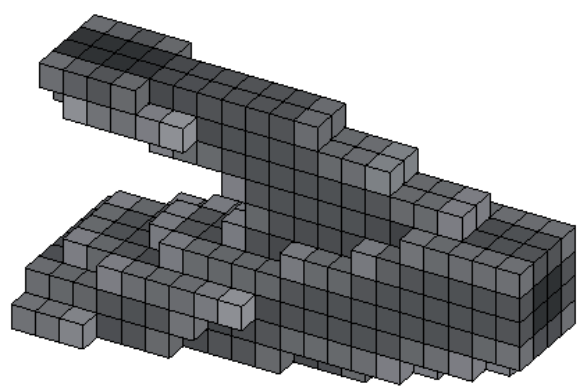

Fig. 20 The exact optimal shape of the 3D cantilever beam

The Matlab code of the volume constrained expected compliance minimization problem can be developed, for example, by straightforward modifications from the top3d.m program presented by Liu and Tovar [2] for 3D continuum structures. In the program, similarly to the 2D case, only two labelled code sections have to be modified to get a code for the exact volume constrained expected compliance minimization with minimal programming effort. The first code section which is labelled by "응 USER DEFINED LOAD DOFs" defines the loads and supports and the second section labelled by "\% OBJECTIVE FUNCTION AND SENSITIVITY ANALYSIS" defines the objective function and its sensitivities. The problem-specific code section replacements for the first 3D example are the followings:

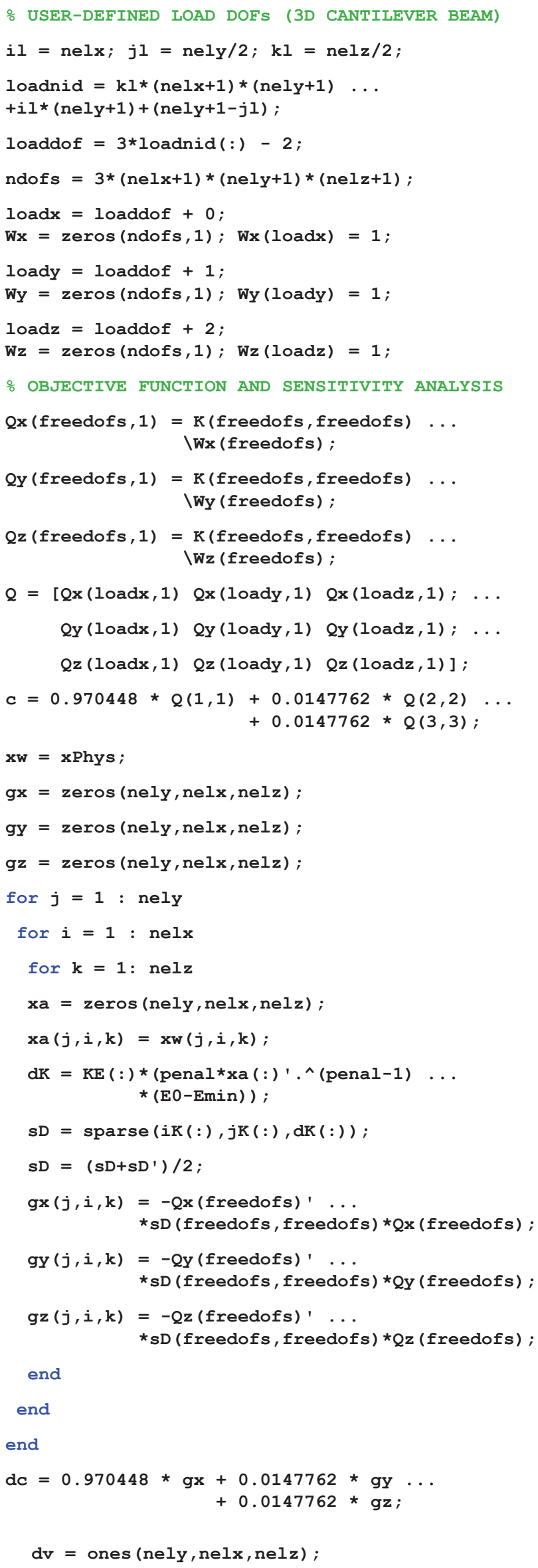

The Mathematica and Matlab codes for the two other loading scenarios can be given by very simple modifications from the presented 3D codes of the first example. 
It may be interesting to mention that in Mathematica the explicit product of two or more probability density functions (PDFs) can be replaced by a "more elegant" nested PDF [ ProductDistribution [...] ...] statement combination.

The presented correctly described benchmark problems with reproducible exact numerical results may be used for testing the quality and computational efficiency of exact and heuristic solution procedures to be developed in the future for structural optimization of continuum structures with varying load directions.

\section{Conclusions}

In this paper, it was shown that when the numerical values of the statistical parameters of a volume-constrained expected compliance minimization problem are known then it can be solve as a volume-constrained single-load topology optimization problem where the objective is the exact analytical expected compliance function in a symbolically and automatically generated extremely simple form. In this study, Mathematica was used to symbolically integrate and simplify the highly nonlinear expected compliance function. It was demonstrated by examples that the result of the symbolic pre-processing step will be a linear function defined on a particular subset of the inverse stiffness entries needed in the compliance computation. The coefficient set of this linear function forms the base of the exact analytical gradient computation used in the optimal solution searching OC method to define the best descent direction. Matlab codes of the algorithm for 2D and 3D structures with exact analytical sensitivities have been developed using the topology optimization codes presented by Andreassen et al. [1] for 2D and Liu and Tovar [2] for 3D structures as starting points. Illustrative examples using the SIMP type topology optimization procedure were presented to demonstrate the essence and viability of the proposed approach and highlight the potential of automatic symbolic computation in structure optimization.

\section{Acknowledgments}

The present study was supported by the Hungarian National Scientific and Research Foundation (OTKA) (grant K 119440).

\section{References}

[1] Andreassen, E., Clausen, A., Schevenels, M., Lazarov, B.S., Sigmund, O. „Efficient topology optimization in MATLAB using 88 lines of code”. Structural and Multidisciplinary Optimization. 43 (1), pp. 1-16. 2011. DOI: 10.1007/s00158-010-0594-7

[2] Liu, K., Tovar, A. “An efficient 3D topology optimization code written in Matlab". Structural and Multidisciplinary Optimization. 50 (6), pp. 1175 1196. 2014. DOI: 10.1007/s00158-014-1107-x

[3] Bendsøe, M. P., Sigmund, O. "Topology Optimization: Theory, Methods and Applications". 370 p. Springer-Verlag, Berlin, Heidelberg. 2004. DOI: 10.1007/978-3-662-05086-6

[4] Deaton, J. D., Grandhi, R. V. “A survey of structural and multidisciplinary continuum topology optimization: post 2000". Structural and Multidisciplinary Optimization. 49 (1), pp. 1-38. 2014. DOI: 10.1007/ s00158-013-0956-Z
[5] Ben-Tal, A., El Ghaoui, L., Nemirovski, A. S. "Robust Optimization". 576 p. Princeton Series in Applied Mathematics. Princeton University Press. 2009. http://press.princeton.edu/titles/9099.html

[6] Csébfalvi, A., Lógó, J. “Critical Examination of Volume-Constrained Topology Optimization for Uncertain Load Magnitude and Direction". In: Proc of ICCSEEC 15. Civil-Comp Press, Stirlingshire, UK. 2015. DOI: $10.4203 /$ ccp.108.189

[7] De Gournay, F., Allaire, G., Jouve, F. "Shape and topology optimization of the robust compliance via the level set method". ESAIM: Control, Optimisation and Calculus of Variations. 14 (1), pp. 43-70. 2008. DOI: 10.1051/cocv:2007048

[8] Thore, C. J., Holmberg, E., Klarbring, A. "Large-scale robust topology optimization under load-uncertainty". In: Proc of 11th World Congress on Structural and Multidisciplinary Optimization, Sydney, Australia, June 7-12，2015. http://web.aeromech.usyd.edu.au/WCSMO2015/ papers/1096_paper.pdf

[9] Csébfalvi, A. "Structural optimization under uncertainty in loading directions: benchmark results". Advances in Engineering Software. In press, 2016. DOI: 10.1016/j.advengsoft.2016.02.006

[10] Dunning, P. D., Kim, H. A., Mullineux, G. "Introducing Loading Uncertainty in Topology Optimization”. AIAA Journal. 49 (4), pp. 760 768. 2011. DOI: 10.2514/1.J050670

[11] Lógó, J. "SIMP type topology optimization procedure considering uncertain load position". Periodica Polytechnica-Civil Engineering. 56 (2), pp. 213-219. 2012. DOI: 10.3311/pp.ci.2012-2.07

[12] Carrasco, M., Ivorra, B., Lecaros, R., Ramos, A. M. “An expected compliance model based on topology optimization for designing structures submitted to random loads". Differential Equations \& Applications. 4 (1), pp. 111-120. 2012. http://files.ele-math.com/articles/dea-04-07.pdf

[13] Liu, J., Wen, G. "Topology optimization of continuum structures with uncertainty in loading direction”. In: Proc of M2D, Ponta Delgada, Portugal, 2015.

[14] Guo, X., Zhao, X., Zhang, W., Yan, J., Sun, G. "Multi-scale robust design and optimization considering load uncertainties". Computer Methods in Applied Mechanics and Engineering. 283, pp. 994-1009. 2015. DOI: 10.1016/j.cma.2014.10.014

[15] Sigmund, O. "A 99 line topology optimization code written in Matlab". Structural and Multidisciplinary Optimization. 21 (2), pp. 120-127. 2001. DOI: $10.1007 / \mathrm{s} 001580050176$

[16] Alvarez, F., Carrasco, M. "Minimization of the expected compliance as an alternative approach to multiload truss optimization". Structural and Multidisciplinary Optimization. 29 (6), pp. 470-476. 2005. DOI: 10.1007/ s00158-004-0488-7

[17] Carrasco, M., Ivorra, B., Ramos, A. M. "Stochastic topology design optimization for continuous elastic materials". Computer Methods in Applied Mechanics and Engineering. 289, pp. 131-154. 2015. DOI: 10.1016/j.cma.2015.02.003

[18] Dunning, P. D., Kim, H. A. "Robust Topology Optimization: Minimization of Expected and Variance of Compliance". AIAA Journal. 51(11), pp. 2656-2664. 2013. DOI: 10.2514/1.J052183

[19] Richardson, J. N., Coelho, R. F., Adriaenssens, S. "Robust topology optimization of 2D and 3D continuum and truss structures using a spectral stochastic finite element method". In: Proc of WCSMO 10, Orlando, Florida, USA. 2013. http://www2.mae.ufl.edu/mdo/Papers/5360.pdf

[20] Zhao, J., Wang, C. "Robust topology optimization under loading uncertainty based on linear elastic theory and orthogonal diagonalization of symmetric matrices". Computer Methods in Applied Mechanics and Engineering. 273, pp. 204-218. 2014. DOI: 10.1016/j.cma.2014.01.018 